\title{
High-resolution structure and reaction cycle of Fatty Acid Photodecarboxylase: anatomy of a crime scene
}

\section{Sorigué ${ }^{1}$, K. Hadjidemetriou ${ }^{2}$, S. Blangy ${ }^{1}$, G. Gotthard ${ }^{3}$, P. Legrand ${ }^{4}$, D. Nurizzo ${ }^{3}$, A. Royant ${ }^{3}$, C. Berthomieu ${ }^{1}$, M. Weik ${ }^{2}$, T. Domratcheva ${ }^{5}$, K. Brettel ${ }^{6}$, M. H. Vos $^{7}$, I. Schlichting ${ }^{8}$, P. Müller ${ }^{7}$, F. Beisson ${ }^{1}$, P. Arnoux ${ }^{1}$}

${ }^{1} A M U, C E A, C N R S$, BIAM Cadarache, 13108 Saint-Paul-lez-Durance, France, ${ }^{2} U G A, C E A, C N R S$, Institut de Biologie Structurale, 38000 Grenoble, France, ${ }^{3}$ ESRF, 38043 Grenoble, France, ${ }^{4}$ SOLEIL, 91192 Gif-sur-Yvette, France, ${ }^{5}$ Department of Chemistry,

Lomonosov Moscow State University, Moscow 119991, Russia, ${ }^{6}$ Université Paris-Saclay, CEA, CNRS, I2BC, 91198 Gif-sur-Yvette, France, ${ }^{7}$ LOB, CNRS, INSERM, Ecole Polytechnique, 91128 Palaiseau, France, ${ }^{8}$ MPI, 69120 Heidelberg, Germany.

$$
\text { pascal.arnoux@cea.fr }
$$

Fatty Acid Photodecarboxylase (FAP) is a recently discovered photoenzyme that catalyzes the conversion of fatty acids into alkane and $\mathrm{CO}_{2}$ under light, with potential importance in green chemistry applications [1]. Its mechanism was still not fully understood and partly relied on a low-resolution crystal structure obtained from crystals with a twinning default [1]. Here, we present high-resolution crystal structures of FAP obtained in the dark and after light illumination at cryogenic temperatures (Figure 1). Combined with structural, computational, and spectroscopic techniques we are now able to provide a detailed reaction cycle of FAP. The reaction mechanism starts with an electron transfer from the fatty acid to a photoexcited oxidized flavin cofactor. Decarboxylation yields an alkyl radical, which is then reduced by back electron transfer and protonation rather than hydrogen atom transfer. Along with flavin reoxidation by the alkyl radical intermediate, a major fraction of the cleaved $\mathrm{CO}_{2}$ unexpectedly transforms in 100 ns, most likely into bicarbonate. This is orders of magnitude faster than in solution, which indicates a catalytic step. FT-IR, structural and functional studies on variants centered on two conserved active site residues (R451 and C432) showed that R451 is essential for substrate stabilization and proton transfer. Altogether this study provides a detailed characterization of this unique enzyme and reveals a striking and unanticipated mechanistic complexity [2].
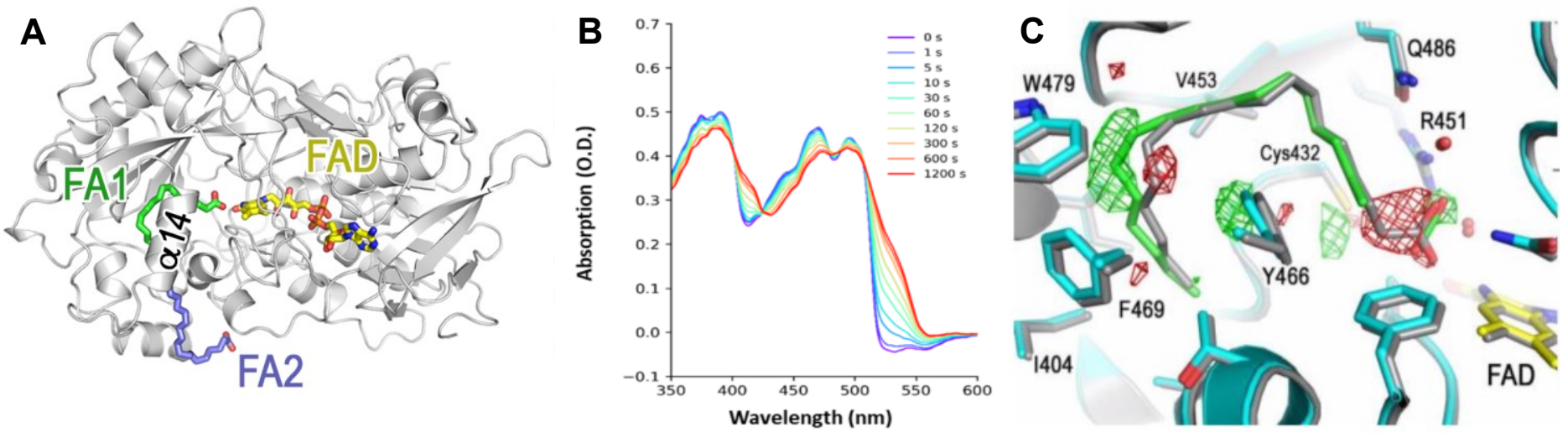

Figure 1. Overall structure of FAP and trapping of a key reaction intermediate at $100 \mathrm{~K}$. (A) Overall structure of FAP, with two substrates stabilized (FA1 and FA2). (B) Building of a key reaction intermediate (FAD in a red-shifted state) along blue-light illumination at $100 \mathrm{~K}$. (C) Experimental difference density map $\left(F_{\text {light }}-F_{\text {dark }}\right)$ contoured at $+/-4 \sigma$ around the active site substrate, superimposed on the structures of the dark state (gray) and the red-shifted form (cyan, with FAD in yellow and alkane/ $\mathrm{CO}_{2}$ in green). $\mathrm{CO}_{2}$ formation is clearly visible, together with the retreat of the alkane product and a rotation of the side chain of Y466.

[1] Sorigué D, Légeret B, Cuiné S, Blangy S, Moulin S, Billon E, Richaud P, Brugière S, Couté Y, Nurizzo D, Müller P, Brettel K, Pignol D, Arnoux P, Li-Beisson Y, Peltier G, Beisson F. (2017) Science. 357, 903.

[2] Sorigué, D., K. Hadjidemetriou, S. Blangy, G. Gotthard, A. Bonvalet, N. Coquelle, P. Samire, A. Aleksandrov, L. Antonucci, A. Benachir, S. Boutet, M. Byrdin, M. Cammarata, S. Carbajo, S. Cuiné, R. B. Doak, L. Foucar, A. Gorel, M. Grünbein, E. Hartmann, R. Hienerwadel, M. Hilpert, M. Kloos, T. J. Lane, B. Légeret, P. Legrand, Y. Li-Beisson, S. L. Y. Moulin, D. Nurizzo, G. Peltier, G. Schirò, R. L. Shoeman, M. Sliwa, X. Solinas, B. Zhuang, T. R. M. Barends, J.-P. Colletier, M. Joffre, A. Royant, C. Berthomieu, M. Weik, T. Domratcheva, K. Brettel, M. H. Vos, I. Schlichting, P. Arnoux, P. Müller, F. Beisson (2021) Science 372, 148.

\section{Keywords: Photoenzyme; Fatty Acid Photodecarboxylase; Reaction mechanism; Alkane biosynthesis}

